\title{
ENZIMAS FIBROLÍTICAS EXÓGENAS EN LA DIGESTIBILIDAD IN VITRO DE CINCO ECOTIPOS DE Brachiaria ${ }^{1}$
}

\author{
Juan H. Avellaneda-Cevallos ${ }^{2}$, Sergio S. González-Muñoz ${ }^{3}$, Juan M. Pinos-Rodríguez ${ }^{4}$, \\ Alfonso Hernández-Garay ${ }^{3}$, Oziel Dante Montañez-Valdez ${ }^{5}$, José Ayala-Oseguera ${ }^{6}$
}

\section{RESUMEN}

Enzimas fibrolíticas exógenas en la digestibilidad in vitro de cinco ecotipos de Brachiaria. Este experimento se llevó a cabo en la Estación Experimental del Colegio de Postgraduados, en Montecillo, Estado de México, México, durante el 25 abril y el 27 noviembre de 2002. Se usó la primera fase de la técnica de Tilley y Terry $(3,6,12,24,48,72 \mathrm{~h}$ incubación) con el objetivo de medir el efecto de un compuesto enzimático fibrolítico exógeno (enzima; Fibrozyme ${ }^{\circledR} ; 0$ y $1,5 \mathrm{~g}$ enzima/kg MS) en la digestibilidad in vitro (DIV) de MS, FDN y FDA de henos de cinco ecotipos de Brachiaria [brizantha var. Toledo (BT); ruziziensis x decumbens CIAT 46024 (RD); decumbens var. Señal (DS); ruziziensis x brizantha CIAT 36061 cv. Mulato (RBM); brizantha var. Insurgente (BI)]. La DIVMS a las 48 y $72 \mathrm{~h}$, para 0 y $1,5 \mathrm{~g}$ enzima, fue mayor $(\mathrm{p}<0,05)$ para los ecotipos BT y BI, respecto a RD, DS y RBM. La DIVFDN a las 48 y $72 \mathrm{~h}$, para 0 y 1,5 g enzima, fue mayor $(\mathrm{p}<0,05)$ para BT y BI, respecto a RD, DS y RBM. La enzima aumentó $(\mathrm{p}<0,05)$ la DIVFDN sólo para el ecotipo RD a las 72 h; además, incrementó la DIVFDA para BT a 12 h; BT y DS a 24 h; BT, RD y BI a 48 h. Por tanto, las enzimas fibrolíticas aumentaron la digestibilidad in vitro de la pared celular de cinco ecotipos de Brachiaria.

Palabras clave: Digestibilidad, FDN, FDA, compuesto enzimático, Brachiaria sp.

\begin{abstract}
Exogenous fibrolytic enzymes on in vitro digestibility of five Brachiaria cultivars. This experiment was conducted at the Experimental Station the Colegio de Postgraduados, in Montecillo, Estado de México, México, during April 25 and November 27 of 2002. The first phase of the Tilley and Terry technique $(3,6,12,24,48,72 \mathrm{~h}$ incubation) was used with to determine the effect of an exogenous fibrolytic enzymatic compound (Fibrozyme ${ }^{\circledR} ; 0$ and $1.5 \mathrm{~g}$ enzyme $/ \mathrm{kg} \mathrm{DM}$ ) on in vitro digestibility (IVD) of DM, NDF and ADF of hays of five ecotypes of Brachiaria [brizantha var. Toledo (BT); ruziziensis x decumbens CIAT 46024 (RD); decumbens var. Señal (DS); ruziziensis x brizantha CIAT $36061 \mathrm{cv}$. Mulato (RBM); brizantha var. Insurgente (BI)]. The IVDDM at 48 and $72 \mathrm{~h}$, for 0 and $1.5 \mathrm{~g}$ enzyme, was higher $(\mathrm{p}<0.05)$ for ecotypes BT and BI, as compared to RD, DS and RBM. The IVDNDF at 48 and $72 \mathrm{~h}$, for 0 and $1.5 \mathrm{~g}$ enzyme, was higher $(\mathrm{p}<0.05)$ for $\mathrm{BT}$ and $\mathrm{BI}$, as compared to RD, DS and RBM. The enzymatic compound increased the IVDNDF only for ecotype $\mathrm{RD}$ at $72 \mathrm{~h}$. Besides, the enzymatic compound increased the IVDADF for BT at $12 \mathrm{~h}$; BT and DS at $24 \mathrm{~h}$; BT, RD and BI at $48 \mathrm{~h}$. Therefore, the exogenous enzymatic fibrolytic compound increased in vitro digestibility of five Brachiaria ecotypes.
\end{abstract}

Key words: Digestibility, NDF, ADF, enzymatic compound, Brachiaria sp.

1 Recibido: 24 de marzo, 2006. Aceptado: 5 de marzo, 2007 Trabajo elaborado como parte de tesis doctoral.

2 Unidad de Investigación Científica y Tecnológica, Facultad de Ciencias Pecuarias, Universidad Técnica Estatal de Quevedo. Quevedo, Ecuador. Av. Quito. Km 1.5 vía Quevedo-Santo Domingo. Apartado Postal 73. Quevedo, Los Rios, Ecuador. Tel. +593 5 2752177. Correo electrónico: juan_avellaneda@yahoo.com.

3 Colegio de Postgraduados. Programa de Ganadería. Carretera México-Texcoco. Km. 36.5. C.P. 56230. Montecillo, Texcoco, Estado de México, México. Tel. +5959520200 ext. 1715. Correo electrónico: sergiogabriel@colpos.mx y hernan@colpos.mx

4 Instituto de Investigación de Zonas Desérticas, Universidad Autónoma de San Luís Potosí, San Luís Potosí, México. Correo electrónico: jpinos@uaslp.mx

5 División de Bienestar y Desarrollo Regional. Departamento de Desarrollo Regional. CUSUR. Universidad de Guadalajara. Ciudad Guzmán, Municipio de Zapotlán El Grande, Jalisco, México. Tel. +52 34157522 22. Ext. 6085. Correo electrónico: montanez77@ hotmail.com.

6 Departamento de Zootecnia. Universidad Autónoma de Chapingo. Carretera México-Texcoco. Km 38.5 C.P. 56230. Texcoco, Estado de México, México. Correo electrónico: jayala@correo.chapingo.mx 


\section{INTRODUCCIÓN}

Los forrajes representan la fuente más económica para la alimentación de los rumiantes, principalmente en el trópico donde hay grandes extensiones de tierra dedicadas a la explotación ganadera. En casi todos los países tropicales la expansión de la frontera agrícola llegó a su límite, y el crecimiento actual de la actividad agropecuaria depende en alto grado de la intensificación y tecnificación de la producción.

En las zonas tropicales de México se produjeron 1.333.100.000 litros de leche en el año 2000 (SAGARPA 2000) y 542.724 t de carne en el 2001 (Gallardo et al. 2002). El hato ganadero en estas zonas se alimenta principalmente mediante pastoreo en áreas cubiertas por grama nativa y especies introducidas (Gallardo et al. 2002). Por tal razón, en estos ecosistemas es importante que los ganaderos dispongan de opciones forrajeras que aumenten la productividad pecuaria, con un enfoque sostenible. Esto permitirá sustituir o complementar especies poco productivas por otras de mayor rendimiento, y con mejores características agronómicas, con las cuales será posible una producción sostenible de la pradera y del hato ganadero.

En este sentido, el género Brachiaria tiene gran potencial como recurso genético forrajero, ya que tiene gran capacidad de adaptación a una amplia gama de condiciones edáficas y climáticas (Valle 1990, citado por Enríquez y Romero 1999). En la década de 1980, el Instituto de Investigaciones Forestales, Agrícolas y Pecuarias (INIFAP) - México, evaluó varios ecotipos del género Brachiaria de los cuales tres fueron formalmente liberados: $B$. decumbens var. Señal o Chontalpo, $B$. brizantha var. Insurgente y $B$. humidicola var. Chetumal, los cuales resultaron sobresalientes en diversos ecosistemas del trópico mexicano (Enríquez y Peralta 1992, citados por Enríquez y Romero 1999).

Sin embargo, las gramíneas tienen una alta concentración de pared celular $(65-75 \%)$ y baja digestibilidad ( $45-55 \%$ ), factores asociados con una reducción en consumo voluntario y menor calidad nutritiva del forraje (Gallean y Goetsch 1993). Por tal motivo, el objetivo de este experimento fue evaluar el efecto de un compuesto enzimático fibrolítico exógeno en la degradación in vitro de la materia seca y fracciones de la pared celular de cinco ecotipos del género Brachiaria.

\section{MATERIALES Y MÉTODOS}

Esta investigación se realizó en el laboratorio de bromatología del Colegio de Postgraduados ubicado en Montecillo, Estado de México, México entre el 25 de abril y 27 de noviembre del 2002. Se utilizaron cuatro borregos Suffolk $(74 \pm 2 \mathrm{~kg}$ PV) con cánula ruminal y alimentados ad libitum (a libertad) con heno de pasto guinea (Panicum maximum var. Mombasa), premezcla mineral y agua, en la unidad metabólica para rumiantes, del Programa de Ganadería, de la granja experimental del Colegio de Postgraduados, Montecillo, Estado de México, México. Se usaron henos de cinco ecotipos de Brachiaria [(brizantha var. Toledo (BT), ruziziensis $\mathrm{x}$ decumbens CIAT 46024 (RD); decumbens var. Señal (DS); ruziziensis x brizantha CIAT $36061 \mathrm{cv}$. Mulato (RBM); brizantha var. Insurgente (BI)] cortado a una edad de $35 \mathrm{~d}$ y henificado a la intemperie en Ciudad Isla, Estado de Veracruz, México, entre el 14 de octubre de 2001 y 11 de enero de 2002.

El compuesto enzimático fibrolítico exógeno (Fibrozyme®, Alltech INC, Nicholasville, KY, U.S.A) es una combinación de extracto de la fermentación de Aspergillus níger y Trichoderma viride y fermentos solubles, protegidos por técnicas de glucosilación. Su actividad xilanásica es de 100 UI/g (una unidad xilanásica es la cantidad de enzima necesaria para liberar $1 \mu \mathrm{mol}$ de xilosa). En los henos se midió materia seca (MS) y orgánica (MO), proteína total (PT; $\mathrm{N}$ x 6,25) y cenizas (AOAC 1990); fibra detergente neutro (FDN) y ácido (FDA) (Van Soest et al. 1991) (Cuadro 1).

Para la digestibilidad in vitro (DIV) de la MS (DIVMS) se utilizó la primera fase de la técnica de Tilley y Terry (1963), mezclando saliva artificial (McDougall 1948) y líquido ruminal (relación 4:1). Con una bomba de vacío, se tomó un litro de líquido ruminal de cada borrego, se mezcló, se filtró con cuatro capas de gasa y depositó en un termo $\left(39^{\circ} \mathrm{C}\right)$ para ser gaseado con $\mathrm{CO}_{2}$.

El medio (40 ml saliva de McDougall y $10 \mathrm{ml}$ de líquido ruminal) se depositó en tubos de propileno 
Cuadro 1. Composición química $(\%)^{\dagger}$ de heno de pasto Brachiaria cortado a una edad de 35 d. Colegio de Postgraduados, Montecillo, Estado de México, México. 2002.

\begin{tabular}{lcccccc}
\hline & MS & MO & PT & FDN & FDA & Cenizas \\
\hline Toledo & 92,18 & 77,59 & 8,56 & 71,30 & 47,87 & 22,41 \\
CIAT 46024 & 92,24 & 71,10 & 7,87 & 70,71 & 48,12 & 28,90 \\
Señal & 92,77 & 70,85 & 6,96 & 73,40 & 52,69 & 29,15 \\
CIAT 36061 cv, Mulato & 91,59 & 78,45 & 6,47 & 74,28 & 53,97 & 21,55 \\
Insurgente & 91,60 & 77,60 & 8,42 & 71,66 & 49,33 & 22,40 \\
\hline
\end{tabular}

tEn base seca.

IMS: materia seca; MO: materia orgánica; PT: proteína; FDN: fibra detergente neutro; FDA: fibra detergente ácido.

$(100 \mathrm{ml})$ con $500 \mathrm{mg}$ de heno de pasto Brachiaria molido (malla de $1 \mathrm{~mm}$ ) y secado a $60{ }^{\circ} \mathrm{C}$ durante $24 \mathrm{~h}$, sin y con $1,5 \mathrm{~g}$ compuesto enzimático/kg MS de Brachiaria. Los tubos fueron gaseados con $\mathrm{CO}_{2}$, tapados para mezclar su contenido y colocados en baño María $\left(39^{\circ} \mathrm{C}\right)$; la incubación se hizo durante 3, $6,12,24,48$ y $72 \mathrm{~h}$ y fue detenida por congelación $(0$ $\left.{ }^{\circ} \mathrm{C}\right)$. Los tubos fueron descongelados a temperatura ambiente para filtrar su contenido a través de papel filtro $541\left(\varnothing\right.$ poro $10 \mu \mathrm{m}$ ) y luego secados a $60{ }^{\circ} \mathrm{C}$ para medir la DIVMS. En las fracciones de FDN y FDA, la digestibilidad in vitro (DIVFDN y DIVFDA) se determinó según Tilley y Terry (1963).

El diseño experimental fue completamente al azar con un arreglo factorial $5 \times 2$ (cinco ecotipos de Brachiaria; compuesto enzimático 0 y $1,5 \mathrm{~g} / \mathrm{kg}$ MS de Brachiaria) en los seis tiempos de incubación (3, 6, 12, 24, 48, 72 h). Se usaron tres tubos (repeticiones) para cada ecotipo y la prueba de digestibilidad se hizo en dos corridas, para utilizar cada tubo como submuestra e incluir la interacción tratamiento x corrida como término de error en el modelo (Pinos-Rodríguez et al. 2002a). El modelo estadístico utilizado fue el siguiente:

$$
\mathrm{Y}_{i j k l}=\mu+\mathrm{C}_{i}+\mathrm{A}_{j}+\mathrm{B}_{k}+\mathrm{AB}_{j k}+\varepsilon_{i j k l}
$$

Donde:

$\mathrm{Y}_{i j k l}=$ Valor de la variable respuesta en la corrida $\mathrm{i}$, ecotipo de Brachiaria $j, g$ del compuesto enzimático $k$. $\mu=$ Media general

$\mathrm{C}_{i}=$ Efecto de la corrida $i$

$\mathrm{A}_{j}=$ Efecto del ecotipo de Brachiaria $j$

$\mathrm{B}_{k}=$ Efecto de $g$ del compuesto enzimático $k$

$\mathrm{AB}_{j k}=$ Efecto de la interacción ecotipo de Brachiaria

$j$ y $g$ del compuesto enzimático $k$

$\varepsilon_{i j k l}=$ Error experimental

Los datos se analizaron usando el procedimiento GLM del paquete estadístico SAS (1999) y las diferencias de medias fueron comparadas usando la prueba de Tukey $(\mathrm{p}<0,05)$.

\section{RESULTADOS Y DISCUSIÓN}

La DIVMS no fue diferente $(\mathrm{p}>0,05)$ entre los ecotipos durante las primeras $24 \mathrm{~h}$ de incubación (Cuadro 2), probablemente porque ese tiempo no es suficiente para cambiar esta variable u observar posibles cambios. Este resultado concuerda con Avellaneda-Cevallos et al. (2003), quienes no encontraron diferencias en la DIVMS de heno de pasto guinea (Panicum maximum var. Mombasa) de 35 y 90 d durante las primeras 48 h de incubación.

La DIVMS de los henos de los ecotipos DS y RBM fue menor $(\mathrm{p}<0,05)$ respecto a BT y BI a las 48 y $72 \mathrm{~h}$; mientras que la DIVMS de RD y RBM fue más alta $(\mathrm{p}<0,05)$ que la de DS a las $72 \mathrm{~h}$. Estos resultados concuerdan con Herrero et al. (2001) 
Cuadro 2. Efecto del compuesto enzimático fibrolítico exógeno en la desaparición (\%) in vitro de MS de heno de cinco ecotipos de Brachiaria. Colegio de Postgraduados, Montecillo, Estado de México, México. 2002.

\begin{tabular}{|c|c|c|c|c|c|c|c|c|c|c|c|}
\hline \multirow{2}{*}{$\begin{array}{c}\text { Incubación } \\
(\mathrm{h}) \\
\end{array}$} & \multicolumn{2}{|c|}{ Toledo (BT) } & \multicolumn{2}{|c|}{ CIAT 46024 (RD) } & \multicolumn{2}{|c|}{ Señal (DS) } & \multicolumn{2}{|c|}{$\begin{array}{c}\text { CIAT } 36061 \\
\text { cv. Mulato (RBM) } \\
\end{array}$} & \multicolumn{2}{|c|}{ Insurgente (BI) } & \multirow[b]{2}{*}{ EEM } \\
\hline & $0,0^{£}$ & 1,5 & 0,0 & 1,5 & 0,0 & 1,5 & 0,0 & 1,5 & 0,0 & 1,5 & \\
\hline 3 & $20,77 \mathrm{a}$ & $20,02 \mathrm{a}$ & $15,04 \mathrm{abc}$ & $15,10 \mathrm{abc}$ & $10,99 \mathrm{c}$ & $13,93 \mathrm{abc}$ & $12,92 \mathrm{bc}$ & $13,00 \mathrm{abc}$ & $16,50 \mathrm{abc}$ & $15,61 \mathrm{abc}$ & 1,27 \\
\hline 6 & $21,65 \mathrm{a}$ & $21,96 a$ & $18,57 \mathrm{a}$ & $20,12 \mathrm{a}$ & $15,17 \mathrm{a}$ & $17,26 \mathrm{a}$ & $13,91 \mathrm{a}$ & $14,94 \mathrm{a}$ & $19,81 \mathrm{a}$ & $18,27 \mathrm{a}$ & 1,52 \\
\hline 12 & $23,89 \mathrm{ab}$ & $25,42 \mathrm{a}$ & $20,42 \mathrm{ab}$ & $21,67 \mathrm{ab}$ & $21,13 \mathrm{ab}$ & $20,88 \mathrm{ab}$ & $17,92 b$ & $19,70 \mathrm{ab}$ & $25,69 \mathrm{a}$ & $21,61 \mathrm{ab}$ & 1,07 \\
\hline 24 & $33,73 a$ & $32,63 a$ & $30,49 a b c$ & $28,73 a b c$ & $24,95 \mathrm{bc}$ & $25,86 b c$ & $24,50 \mathrm{c}$ & $25,44 \mathrm{bc}$ & $34,00 \mathrm{a}$ & $31,26 a b$ & 1,14 \\
\hline 48 & $50,24 \mathrm{a}$ & $49,36 \mathrm{a}$ & $42,71 \mathrm{bcd}$ & $41,04 \mathrm{cde}$ & $37,45 \mathrm{e}$ & $36,63 \mathrm{e}$ & $37,15 \mathrm{e}$ & $39,15 \mathrm{ed}$ & $47,14 \mathrm{ab}$ & $45,61 \mathrm{abc}$ & 0,87 \\
\hline 72 & $58,22 \mathrm{a}$ & $56,18 \mathrm{a}$ & $49,41 b$ & $50,01 \mathrm{~b}$ & $43,56 \mathrm{c}$ & $43,67 \mathrm{c}$ & $48,47 b$ & $49,44 b$ & $57,05 \mathrm{a}$ & $55,82 \mathrm{a}$ & 0,51 \\
\hline
\end{tabular}

a, b, c, d, e Medias con diferentes letras en una hilera, son diferentes $(\mathrm{p}<0,05)$.

£ g compuesto enzimático/kg MS brachiarias

I EEM: error estándar de la media.

quienes reportaron diferencias en la DIVMS entre varios ecotipos de Brachiaria, aunque B. ruziziensis es más digerible, seguida por $B$. decumbens, mientras que $B$. brizantha y $B$. humidicola tiene la menor digestibilidad. Esto último difiere con lo observado en la presente investigación, ya que la digestibilidad de los henos de los ecotipos de brizantha presentaron mejor $(\mathrm{p}<0,05)$ digestibilidad que los de $B$. decumbens. Tal diferencia puede deberse a factores como características agronómicas de los ecotipos, efectos climáticos, recolección de las muestras y su manejo.

La DIVMS para el heno del ecotipo DS fue menor $(p<0,05)$ en 23,$7 ; 12,3 ; 10,91$ y $22,7 \%$, cuando se comparó con BT, RD, RBM y BI.

El compuesto enzimático no mejoró $(\mathrm{p}>0,05)$ la DIVMS de los ecotipos de Brachiaria (Cuadro 2). De manera similar, el tratamiento con un compuesto enzimático no cambió la DIVMS en heno de trigo a las $24 \mathrm{~h}$ (Pollard et al. 2001), en heno de pasto guinea (Panicum maximum var. Mombasa) de 3 a 72 h (Avellaneda-Cevallos et al. 2003), en festuca (Festuca arundinacea) después de $18 \mathrm{~h}$ (Tricarico et al. 1998), en heno de alfalfa (Medicago sativa) de 0 a 36 h (Titi et al. 1998), ni en heno de pasto ballico (Lolium perenne) a las 48 y $72 \mathrm{~h}$, y de alfalfa a las 24 y 72 h (Pinos et al. 2001). Sin embargo, el uso de un compuesto enzimático ha mejorado la DIVMS de heno de alfalfa (Pollard et al. 2001) y de festuca en las primeras 12 h (Tricarico et al. 1998); además, según Pinos et al. (2001), aumentó la DIV de la MO del heno de ballico (48 h) y de alfalfa (24, 48 y 72 h). En este caso, las diferencias en los resultados puede deberse a factores como tipo y manejo de las muestras de forraje, así como técnicas y equipo de laboratorio empleados para medir la digestibilidad in vitro.

La DIVFDN (Cuadro 3) a las 48 y $72 \mathrm{~h}$ para los henos de los ecotipos BT y BI fue mayor $(\mathrm{p}<0,05)$ respecto a RD, DS y RBM; además, el compuesto enzimático aumentó $(\mathrm{p}<0,05)$ la DIVFDN a las $72 \mathrm{~h}$ del heno de RD. Lo anterior se puede deber, en parte, a diferencias entre componentes de la pared celular de los ecotipos, y porque las enzimas del compuesto requieren varias horas para cambiar la digestibilidad de la FDN. En los demás tiempos y ecotipos no hubo diferencia $(p>0,05)$ entre el testigo (sin compuesto enzimático) y la adición de $1,5 \mathrm{~g}$ del compuesto enzimático (Cuadro 3). Al respecto, AvellanedaCevallos et al. (2003) observaron incrementos en la DIVFDN $(\mathrm{p}<0,05)$ a las 3,6 y 12 h sólo para heno de guinea (Panicum maximum var. Mombasa) de 90 $\mathrm{d}$, aunque a las 24,48 y $72 \mathrm{~h}$ la DIVFDN fue mayor $(\mathrm{p}<0,05)$ para guinea de $35 \mathrm{~d}$; además, la enzima $(1,5$ g) aumentó $(\mathrm{p}<0,05)$ la DIVFDN para guinea de $35 \mathrm{~d}$ (a las 48 h) y de $90 \mathrm{~d}$ (a las 72 h). Mandebvu et al. (1999) encontraron que la DIVFDN fue mayor $(\mathrm{p}<0,05)$ para un pasto bermuda (Cynodon dactylon) cosechado cada $21 \mathrm{~d}$ comparado con otro cosechado cada $42 \mathrm{~d}$, pero 
Cuadro 3. Efecto del compuesto enzimático fibrolítico exógeno en la desaparición (\%) in vitro de FDN de heno de cinco ecotipos de Brachiaria. Colegio de Postgraduados, Montecillo, Estado de México, México. 2002.

\begin{tabular}{|c|c|c|c|c|c|c|c|c|c|c|c|}
\hline \multirow{2}{*}{$\begin{array}{l}\text { Incubación } \\
\text { (h) }\end{array}$} & \multicolumn{2}{|c|}{ Toledo (BT) } & \multicolumn{2}{|c|}{ CIAT 46024 (RD) } & \multicolumn{2}{|c|}{ Señal (DS) } & \multicolumn{2}{|c|}{$\begin{array}{c}\text { CIAT } 36061 \\
\text { cv. Mulato (RBM) }\end{array}$} & \multicolumn{2}{|c|}{ Insurgente (BI) } & \multirow[b]{2}{*}{ EEM } \\
\hline & $0,0^{£}$ & 1,5 & 0,0 & 1,5 & 0,0 & 1,5 & 0,0 & 1,5 & 0,0 & $\overline{1,5}$ & \\
\hline 3 & $17,92 \mathrm{ab}$ & $17,14 \mathrm{ab}$ & $14,86 \mathrm{~b}$ & $17,59 \mathrm{ab}$ & $17,76 \mathrm{ab}$ & $18,07 \mathrm{ab}$ & $15,56 \mathrm{ab}$ & $17,47 \mathrm{ab}$ & $18,09 \mathrm{ab}$ & $18,77 \mathrm{a}$ & 0,64 \\
\hline 6 & $18,20 \mathrm{a}$ & $18,91 \mathrm{a}$ & $18,07 \mathrm{a}$ & $22,19 a$ & $18,56 \mathrm{a}$ & $19,74 a$ & $17,60 \mathrm{a}$ & $17,13 a$ & $21,49 a$ & $22,74 a$ & 1,03 \\
\hline 12 & $19,23 a$ & $22,79 a$ & $20,65 a$ & $18,64 \mathrm{a}$ & $19,91 \mathrm{a}$ & $19,61 \mathrm{a}$ & $18,51 \mathrm{a}$ & $19,64 \mathrm{a}$ & $22,57 \mathrm{a}$ & $22,71 \mathrm{a}$ & 0,89 \\
\hline 24 & $29,49 \mathrm{ab}$ & $28,80 \mathrm{ab}$ & $27,24 b c$ & $29,00 \mathrm{abc}$ & $26,00 \mathrm{bc}$ & $26,69 b c$ & $25,34 \mathrm{c}$ & $26,12 b c$ & $32,00 \mathrm{a}$ & $33,07 \mathrm{a}$ & 0,71 \\
\hline 48 & $45,68, a b c$ & $45,10 b c$ & $37,15 \mathrm{e}$ & 41,70 cde & $38,18 \mathrm{de}$ & $43,05 \mathrm{cde}$ & $39,51 \mathrm{de}$ & $41,42 \mathrm{cde}$ & $47,44 a b$ & $51,03 \mathrm{a}$ & 0,93 \\
\hline 72 & $58,25 \mathrm{a}$ & $58,29 a$ & $42,41 \mathrm{e}$ & $49,26 \mathrm{bcd}$ & $42,80 \mathrm{de}$ & $44,64 \mathrm{cde}$ & $49,57 b c$ & $50,94 \mathrm{bc}$ & $54,73 \mathrm{ab}$ & $58,49 \mathrm{a}$ & 1,14 \\
\hline
\end{tabular}

a, b, c, d, e Medias con diferentes letras en una hilera, son diferentes $(\mathrm{p}<0,05)$,

${ }^{£} \mathrm{~g}$ enzima/kg MS brachiarias

I EEM: error estándar de la media.

no hubo efecto de un compuesto enzimático exógeno. Sin embargo, según Pinos et al. (2002b), el compuesto enzimático incrementó $(\mathrm{p}<0,05)$ la DIVFDN de heno de alfalfa y de pasto ballico.

La DIVFDA (Cuadro 4) fue mayor $(\mathrm{p}<0,05)$ a las tres horas para los henos de los ecotipos BT, RBM y BI comparados con el RD. Además, el compuesto enzimático aumentó $(\mathrm{p}<0,05)$ la DIVFDA para BT (12, 24 y 48 h), DS (24 h), RBM (48 h), BI (48 h). Al respecto, la DIVFDA de heno de guinea de $35 \mathrm{~d}$, a las 3 , $6,12,24,48$ y 72 , y de guinea de $90 \mathrm{~d}$ a las 24 y $72 \mathrm{~h}$ fue mayor $(\mathrm{p}<0,05)$ cuando se usó el compuesto enzimático (Avellaneda-Cevallos et al. 2003). No obstante, PinosRodríguez et al. (2002b) no observaron diferencias en la digestibilidad in vitro de la FDA de heno de alfalfa y de pasto ballico, al agregar un compuesto enzimático fibrolítico exógeno. La diferencia entre los resultados de este experimento y los publicados por otros investigadores puede deberse al tipo de forraje.

Cuadro 4. Efecto del compuesto enzimático fibrolítico exógeno en la desaparición (\%) in vitro de FDA de heno de cinco ecotipos de Brachiaria. Colegio de Postgraduados, Montecillo, Estado de México, México. 2002.

\begin{tabular}{|c|c|c|c|c|c|c|c|c|c|c|c|}
\hline \multirow{2}{*}{$\begin{array}{c}\text { Incubación } \\
(\mathrm{h})\end{array}$} & \multicolumn{2}{|c|}{ Toledo (BT) } & \multicolumn{2}{|c|}{ CIAT 46024 (RD) } & \multicolumn{2}{|c|}{ Señal (DS) } & \multicolumn{2}{|c|}{$\begin{array}{c}\text { CIAT } 36061 \\
\text { cv. Mulato (RBM) } \\
\end{array}$} & \multicolumn{2}{|c|}{ Insurgente (BI) } & \multirow[b]{2}{*}{ EEM } \\
\hline & $0,0^{\mathfrak{f}}$ & 1,5 & 0,0 & 1,5 & 0,0 & 1,5 & 0,0 & 1,5 & 0,0 & 1,5 & \\
\hline 3 & $6,00 \mathrm{abc}$ & $9,08 \mathrm{a}$ & $0,31 d$ & $-2,09 d$ & $3,02 \mathrm{bcd}$ & $1,62 \mathrm{~cd}$ & $7,55 \mathrm{ab}$ & $10,48 \mathrm{a}$ & $9,80 \mathrm{a}$ & $9,54 \mathrm{a}$ & 0,95 \\
\hline 6 & $7,75 \mathrm{abc}$ & $9,96 a b c$ & $1,95 \mathrm{c}$ & $3,10 \mathrm{bc}$ & $3,78 \mathrm{abc}$ & $9,37 \mathrm{abc}$ & $9,65 \mathrm{abc}$ & $12,55 \mathrm{a}$ & $4,58 \mathrm{abc}$ & $11,34 \mathrm{ab}$ & 1,54 \\
\hline 12 & $7,52 \mathrm{~cd}$ & $14,73 \mathrm{ab}$ & $3,33 \mathrm{~d}$ & $6,88 \mathrm{~cd}$ & $10,03 \mathrm{bc}$ & $12,73 \mathrm{ab}$ & $14,32 \mathrm{ab}$ & $16,58 \mathrm{a}$ & $15,16 \mathrm{a}$ & $14,27 \mathrm{ab}$ & 0,88 \\
\hline 24 & $12,80 \mathrm{~cd}$ & $21,35 \mathrm{ab}$ & $12,70 \mathrm{~cd}$ & $15,28 \mathrm{bcd}$ & $11,42 d$ & $19,49 \mathrm{abc}$ & $19,42 \mathrm{abc}$ & $21,69 a b$ & $22,50 \mathrm{a}$ & $21,76 \mathrm{ab}$ & 1,22 \\
\hline 48 & $32,43 \mathrm{de}$ & $39,21 b$ & $21,07 \mathrm{~g}$ & $28,68 \mathrm{ef}$ & $25,12 f g$ & $28,19 \mathrm{ef}$ & $32,49 \mathrm{cde}$ & $37,85 \mathrm{bcd}$ & $38,85 \mathrm{bc}$ & $45,62 \mathrm{a}$ & 1,11 \\
\hline 72 & $43,47 \mathrm{abc}$ & $48,42 \mathrm{a}$ & $30,69 d$ & $36,11 \mathrm{bcd}$ & $34,22 \mathrm{~cd}$ & $34,30 \mathrm{~cd}$ & $41,56 \mathrm{abc}$ & $44,14 a b c$ & $45,97 \mathrm{ab}$ & $48,42 \mathrm{a}$ & 1,80 \\
\hline
\end{tabular}

$\mathrm{a}, \mathrm{b}, \mathrm{c}, \mathrm{d}, \mathrm{e}, \mathrm{f}$ Medias con diferentes letras en una hilera, son diferentes $(\mathrm{p}<0,05)$.

$£ \mathrm{~g}$ enzima/kg MS brachiarias

I EEM: error estándar de la media. 


\section{CONCLUSIONES}

Con base a las condiciones experimentales descritas, se concluye que los henos de los ecotipos de $B$. brizantha presentaron mejor DIVMS (24, 48 y 72 h) y DIVFDN (48 y 72 h) comparados con los de B. decumbens.

El compuesto enzimático aumentó la DIVFDN para FDN a las $72 \mathrm{~h}$ del heno de ruziziensis $\mathrm{x}$ decumbens CIAT 46024 (RD); en los demás tiempos y ecotipos no hubo cambios en la DIVFDN.

La DIVFDA no fue diferente entre los ecotipos a las $6,12,24,48$ y 72 h. Sin embargo, el compuesto enzimático incrementó la DIVFDA de los ecotipos $B$. brizantha var. Toledo (BT; 12, 24 y 48 h), $B$. decumbens var. Señal (DS; 24 h), B. ruziziensis x $B$. brizantha CIAT $36061 \mathrm{cv}$. Mulato (RBM; (48 h), $B$. brizantha var. Insurgente (BI: $48 \mathrm{~h}$ ).

De acuerdo con estos resultados experimentales, el compuesto enzimático fibrolítico exógeno empleado tuvo efectos positivos en la degradación in vitro de algunas fracciones de la pared celular, principalmente de la FDA, en los henos de los cinco ecotipos de pasto Brachiaria.

\section{LITERATURA CITADA}

AOAC (Association of Official Analytical Chemists). 1990. Official methods of analysis (15 ed.). Washington, DC. 1298 p.

AVELLANEDA-CEVALLOS，J.H.; GONZÁLEZ，S.S.; PINO-RODRÍGUEZ, J.M.; HERNÁNDEZ, A.; BARCENA, R.; COBOS, M.; HERNÁNDEZSÁNCHEZ, D.; MONTANEZ-VALDEZ, O. 2003. Effect of exogenous fibrolytic enzymes (Fibrozyme) on dry matter and cell wall in vitro digestibility of guinea grass (Panicum maximum var. Mombasa) hay. J. Animal Sci (Suppl. 1):334 (Abstr.).

ENRÍQUEZ, J. F.; ROMERO, J. 1999. Tasa de crecimiento estacional a diferentes edades de rebrote de 16 ecotipos de Brachiarias spp. en Isla, Veracruz. Agrociencia. 33:141-148.
GALLARDO, N.J.L.; GARCÍA, C.M.; ALBARRAN, M.; OCHOA, R.; ORTEGA, C. 2002. Situación actual de la producción de carne de bovino en México. Claridades Agropec. 109:3-28.

GALYEAN, M. L.; GOETSCH, A. L. 1993. Utilization of forage fiber by ruminants. In: H. Jung, D. Buxton, R. Hatfield and J. Ralph. eds. Forage Cell Wall Structure and Digestibility. American Society of Agronomy, Crop Science of America, Soil Science Society of America, Madison, Wisconsin, USA. p. 33-71.

HERRERO, M.; DO VALLE, C.B.; HUGHES, N.R.G.; SABATEL, V.; JESSOP, N.S. 2001. Measurements of physical strength and their relationship to the chemical composition of four species of Brachiaria. Anim. Feed Sci. Tech. 92:149-158.

MANDEBVU, P.; WEST, J.W.; FROETSCHEL, M.A.; HATFIELD, R.D.; GATES, R.N.; HILL, G.M. 1999. Effect of enzymes or microbial treatment of Bermudagrass forages before ensiling on cell wall composition, end products of silage fermentation and in situ digestion kinetics. Anim. Feed Sci. Tech. 77:317-329.

McDOUGALL, E.I. 1948. Studies on ruminant saliva. I. The composition and output of sheep's saliva. Biochem. J. 70:99-109.

PINOS, J.M.; GONZÁLEZ, S. S.; MENDOZA, G.; BÁRCENA, R.; COBOS, M. 2001. Efecto de enzimas fibrolíticas glucosiladas en la digestibilidad in vitro de MS y MO de alfalfa (Medicago sativa) y ballico (Lolium perenne). Rev. Fac. Agro. (LUZ) 18:505-509

PINOS-RODRÍGUEZ， J.M.; GONZÁLEZ， S. S.; MENDOZA, G. D.; BÁRCENA, R.; COBOS, M. 2002a. Efecto de enzimas fibrolíticas exógenas en la digestibilidad in vitro de la pared celular de heno de alfalfa (Medicago sativa) o de ballico (Lolium perenne). Interciencia. 27:28-32.

PINOS-RODRÍGUEZ， J.M.; GONZÁLEZ， S. S.; MENDOZA, G. D.; MARTÍNEZ, A. 2002b. Análisis estadístico de experimentos de digestibilidad in vitro con forrajes. Interciencia. 27:143-146. 
POLLARD, G.; WRIGHT, W.; BRAMBL, T.; RICHARDSON, C.; COBB, C. 2001. Effects of liquid feed supplementation and (or) cellulolytic enzymes on dry matter disappearance of either legume or grass hay. J. Dairy Sci. 84 (Suppl. 1):37 (Abstr).

SAGARPA. 2000. Situación actual y perspectivas de la producción de leche de ganado bovino en México 1990-2000. 87 p.

SAS (Statistical Analysis System). 1999. User's guide: Statistics [CD-ROM Computer file]. North Carolina, USA. Version 8. SAS Inst. Inc., Cary, NC.

TILLEY, J.M.; TERRY, R. A. 1963. A two-stage technique for the in vitro digestion of forage crops. J. Brit. Grass. Soc. 28:104-111.
TITI, H.H.; RICHARDSON, C.R.; COBB, C.W. 1998. Effects of fibrolytic enzyme treatment on forage dry matter and organic disappearance. J. Animal Sci. 76 (Suppl. 1):293 (Abstr.).

TRICARICO, J.M.; DAWSON, K.A.; NEWMAN, K. E. 1998. Effects of an exogenous microbial enzyme preparation (Fibrozyme) on ruminal digestion of fescue hay. J. Animal Sci. 76 (Suppl. 1):289 (Abstr.).

VAN SOEST, P.J.; ROBERTSON, J.B.; LEWIS, B.A. 1991. Methods for dietary fiber, neutral detergent fiber, and nonstarch polysaccharides in relation to animal nutrition. J. Anim. Sci. 74:3583-3597. 